\title{
Epidemiological profile of COVID-19 deaths in the public hospital in Rio de Janeiro, Brazil
}

\begin{abstract}
The aim of this study was to describe the epidemiological profiles of the Covid-19 deaths in the public reference hospital Ronaldo Gazolla Hospital ranging from their age, sex, race, cause of death and length of stay until death. Retrospective descriptive observational study with patients who died with a positive COVID-19 diagnosis who presented themselves to this center during the outbreak of April 2020. Our sample included 261 deaths and information reported includes age, sex, and race, cause of death and length of stay until death. All cases were confirmed by diagnostic outcomes, using nasopharyngeal swab samples were collected for extracting 2019-nCoV RNA through the technique of real-time reverse transcription polymerase chain reaction (RT-PCR). Our sample was comprised of $62.1 \%$ men, with a mean age of $60.1(\mathrm{SD}=16.1)$ years. A proportion of $43.3 \%(\mathrm{n}=113)$ of the patients were brown, $16.9 \%(n=42)$ were black and $40.6 \%(n=106)$ were white. The median of duration of hospital stay was 3.8 days $(1.6 ; 6.5)$. Most deaths were caused by severe acute respiratory syndrome $(62.1 \%)$, followed by sepsis $(24.1 \%)$. This study showed that most COVID-19 deaths at the public reference hospital occurred due to severe acute respiratory syndrome (SARS), with a higher proportion of men, brown race and an average age of 60years. The short time of hospital stay between hospitalization and death draws attention to the deficiency of the public health system of Rio de Janeiro city.
\end{abstract}

Keywords: epidemiological profile, COVID-19, severe acute respiratory syndrome
Volume I 3 Issue 5 - 2020

\author{
Alex Souto Maior,' Antônio Assef, ${ }^{2}$ José \\ Carlos de Campos Jr,' Ney Meziat-Filho ${ }^{3}$ \\ 'Department of Physiology, Augusto Motta University Center, \\ Brazil \\ ${ }^{2}$ Department of Medicine, Ronaldo Gazolla Hospital, Brazil \\ ${ }^{3}$ Department of Epidemiology, Augusto Motta University Center, \\ Brazil \\ Correspondence: Alex Souto Maior, Augusto Motta University \\ Centre - UNISUAM Postgraduate Program in Rehabilitation \\ Sciences, Praca das Nacoes, 34 - Bonsucesso, ZIP Code \\ 21041010 - Rio de Janeiro, RJ, Brazil, \\ Email alex.bioegenharia@gmail.com
}

Received: September 15, 2020 | Published: October 30, 2020

\section{Introduction}

Coronavirus is a family of viruses that causes respiratory infections including the new coronavirus (COVID-19) discovered in December of 2019, in Wuhan, China. Corona represents crown-like spikes on the outer surface of the virus; thus, it was named as a Coronavirus. ${ }^{1}$ Full-genome sequencing and phylogenic analysis indicated that 2019$\mathrm{nCoV}$ is a form of betacoronaviruses associated with human severe acute respiratory syndrome (SARS) and Middle East respiratory syndrome (MERS). ${ }^{2,3}$ The COVID-19 is a highly transmittable viral infection and transmitted after contact with infected surfaces and with infected patient's droplets (i.e. saliva and aerosol). ${ }^{3,4}$ Illness ranges in severity from asymptomatic or mild to severe which $80 \%$ of the infected population are either asymptomatic or have mild disease ${ }^{5}$. However, clinical spectrum of coronavirus infection is quite broad, ranging from a simple cold to severe pneumonia. Most infected individuals exhibit signs and symptoms, such as mild respiratory illness, shortness of breath, myalgia, fatigue, and persistent fever, an average of 5-6days after infection (range, 1-14days). ${ }^{2-4}$ On the other hand, severe cases can lead to acute respiratory distress syndrome, arrhythmia, shock, acute cardiac injury, secondary infection, and acute kidney injury, multiple organ failure, and death. ${ }^{2,4,5}$

The COVID-19 outbreak has now spread to more than 190 countries infecting 6,194,533 individuals of all ages, including 376,320 deaths, as of 01 June $2020 .{ }^{6}$ However, the rapid spread throughout the world, the America became the continent with the greatest number of cases among all continents $(2,905,432$ confirmed cases $)$ and 162,701 deaths ${ }^{6}{ }^{6}$ In Brazil, the situation of the epidemic already points to a rising and fast epidemic curve with 526,447 confirmed cases and 29,314 deaths on June 1, 2020..$^{6-8}$ Specifically, in Rio de Janeiro, Brazil, until June $1,2020,54,530$ new cases were confirmed, but the number of deaths seems contradictory without a defined epidemiological profile. ${ }^{7}$ Thus, investigate the epidemiological profile of the patient who died due to complications generated by COVID-19 in the state of Rio de Janeiro becomes important to disseminate critical information among both modelers and policymakers for a timely control response to be implemented. Accordingly, the aim of this report is to describe the epidemiological profiles of the Covid-19 deaths in Ronaldo Gazolla Hospital ranging from their age, sex, and race, cause of death and length of stay until death.

\section{Methods}

\section{Data collection}

Ronaldo Gazolla Hospital is a reference hospital in Rio de Janeiro, Brazil designated for isolation and management of cases of COVID-19. The current study is a retrospective descriptive observational study with patients who died with a positive COVID-19 diagnosis who presented themselves to this center during the outbreak of April 2020. Our sample included 261 deaths and information reported includes age, sex, and race, cause of death and length of stay until death. The clinical outcomes of these patients were monitored during Abril, 2020, the final date of follow-up, when all the patients died. Two research investigators independently reviewed the data collection forms to verify data accuracy. This study was approved by the institutional Ethics Committee for Human Experiments (CAAE: 37048720.8.0000.5235).

\section{Confirmation of COVID- 19 cases}

All cases were confirmed by diagnostic outcomes, using nasopharyngeal swab samples were collected for extracting 2019nCoV RNA through the technique of real-time reverse transcription 
polymerase chain reaction (RT-PCR). ${ }^{9}$ The samples were placed into a collection tube containing virus transport medium (VTM) for extraction of total RNA, the cell lysates were transferred into a collection tube, centrifugated and the suspension was used for RTPCR assay of 2019-nCoVRNA.

\section{Statistical analysis}

The databases obtained were extracted into electronic datasheets and exported to R-project for statistical analysis. Deaths' characteristics were described using proportions; mean and standard deviations; and median and interquartile range (P25; P75) if the data was not normally distributed.

\section{Results}

Our sample was comprised of $62.1 \%$ men, with a mean age of $60.1(\mathrm{SD}=16.1)$ years (Table 1$)$. A proportion of $43.3 \%(\mathrm{n}=113)$ of the patients were brown, $16.9 \%(n=42)$ were black and $40.6 \%(n=106)$ were white (table 1). The median of duration of hospital stay was 3.8 days $(1.6 ; 6.5)$. Most deaths were caused by severe acute respiratory syndrome $(62.1 \%)$, followed by sepsis $(24.1 \%)$ (Table 2$)$.

Table I Characteristics of Covid-19 deaths in the reference hospital

\begin{tabular}{ll}
\hline Variables & Values \\
\hline Age (years); mean (sd) & $60.1(16.1)$ \\
Sex; (n, \%) & \\
Men & $162(62.1)$ \\
Women & $99(37.9)$ \\
Race; (n, \%) & \\
White & $106(40.6)$ \\
Brown & $113(43.3)$ \\
Black & $42(16.9)$ \\
$\begin{array}{l}\text { Duration of hospital stay (days); } \\
\text { median (P25;P75) }\end{array}$ & 3.8 \\
\hline
\end{tabular}

Table 2 Proportions of causes of deaths of COVID-19 patients

\begin{tabular}{ll}
\hline Causes of deaths & N (\%) \\
\hline Severe acute respiratory syndrome (SARS) & $162(62,1 \%)$ \\
Sepsis (shock) & $63(24.1 \%)$ \\
Pneumonia & $13(4.9 \%)$ \\
Hypovolemic shock & $8(3.1 \%)$ \\
Cardiogenic shock & $5(1.9 \%)$ \\
Cardiorespiratory arrest & $2(0,7 \%)$ \\
Cardiac arrhythmia & $2(0,7 \%)$ \\
Multiple organ failure & $\mathrm{I}(0,03 \%)$ \\
Mixed shock & $\mathrm{I}(0,03 \%)$ \\
Pulmonary embolism & $\mathrm{I}(0,03 \%)$ \\
Legal medical institute & $2(0,7 \%)$ \\
Others & $3(1.1 \%)$ \\
\hline
\end{tabular}

\section{Discussion}

The present study investigated indicators and epidemiological profile of patients infected with COVID-19 from hospitalization to death. Our findings showed that most deaths occurred due to severe acute respiratory syndrome (SARS), with the highest proportion of men, brown race and average age of 60years. In addition, we also observed that the length of hospital stay between hospitalization and death was 3.8days.

Few studies were found that evaluated the epidemiological profile between hospitalization and death. SARS was the most frequently observed causes of deaths which the management of the problem is also important in terms of public health. SARS is associated with COVID-19 by clinical features with lymphopaenia, massive infiltration of phagocytes and inflammation sustained by cytokines, reflecting that COVID-19 caused an inflammatory response characterized by deep airway and alveolar damage., ${ }^{40}$ Thus, the lungs, immune organs, and systemic small vessels are the main targets of virus attack, so that extensive consolidation of the lung, diffuse alveolar damage with hyaline membrane formation, respiratory distress, and decreased immune function are the main causes of death. ${ }^{4,10,11}$ On the other hand, present study showed that sepsis occurred in nearly $25 \%$ of adults due to viral infection which results in alveolar and acute respiratory failure. ${ }^{12}$ Thus, some studies show that the main causes of death are related to SARS and sepsis, but the evolution of pulmonary and systemic inflammation in the first and/or second week may determine outcome of disease (death or survival). ${ }^{13-15}$ SARS and Sepsis were the most common complication caused by COVID-19, but further research is needed to investigate the pathogenesis of SARS and sepsis in COVID-19 illness.

COVID-19 show significant host factors for replication which are impacted by age coupled with comorbidities and immune system responses that enhance the severity of the viral infection. ${ }^{16}$ Hence, it has been observed that the alveolar lesions occur in a variety of forms such as exudative inflammation, carnification, interstitial inflammation, fibrosis as well as focal hemorrhage. ${ }^{17}$ Our study showed that deaths due to COVID-19 occurred in patients aged $\geq 60$ years. Corroborating our results, many studies have shown significant mortality rates in China, Germany, Italy, South Korea, Spain and Sweden due to the COVID-19 viral infection in patients aged $\geq 60$ years. ${ }^{13,18,19}$ Epidemiological results have shown that the mortality by COVID-19 viral infection was $<1 \%$ in the age group of $<50$ years, but in the age group of $\geq 60$ years reached values between $16.9 \%$ and $24.4 \%{ }^{19}$ Specifically, in Rio de Janeiro in the period between March 6th and April 10th 2020 the case fatality rate was $26.1 \%$ in patients with age $>60$ years. ${ }^{20}$ The mortality by COVID-19 viral infection in the present study follow the pattern of the international and national data, with greater risk of death for males and people over 60 years old. On the other hand, few modeling studies have examined the case fatality rate mostly because considerable uncertainty exists in regard to the ascertainment rate and asymptomatic rate. ${ }^{5}$

In relation to sex, some studies have shown that men have the highest prevalence rate of COVID-19 infection $(>50 \%) .{ }^{12,15}$ The mortality by COVID-19 viral infection also show high prevalence in men $(>50 \%)$ when compared to women; i.e, a male: female ratio of 2:1. ${ }^{13,20,21}$ Moreover, the random effects model yields an aggregate odds ratio (OR) of 1.84 for comparing the odds of death for males to females, maybe for possible sex differences in the immune response. ${ }^{22}$ The reasons for the observed differences related to COVID-19 deaths are multifactorial. Another important factor is to consider is the stage 
of the epidemic in which the country finds itself. Thus, the data will be useful in determining the treatment policies and preventive measures of COVID-19.

The length of hospital stay between hospitalization and death was very short (median=3.8 $[1.62 ; 6 ; 46])$. There is evidence that the time between the first symptoms of COVID-19 and death ranges between 15 and 20days. ${ }^{23-26}$ This raises the hypothesis that our sample was composed by patients that arrived at the hospital in an advanced stage of COVID-19, since patients were hospitalized on average after 10 days from the beginning of the symptoms, five days before death. There are two reasons that could explain this delay in hospitalization. First, in the beginning of the outbreak in Rio de Janeiro the local public health recommendations were to stay home until shortness of breath. Second, difficulties in the access of public beds in intensive care units (ICU). Goldwassen et al. ${ }^{27}$ showed that the number of ICU beds was not sufficient even before COVID-19 pandemic in Rio de Janeiro state.

The main limitation of the present study was the lack of data of COVID-19 patients that did not die during the period of the study, precluding the calculation of the fatality rate and the investigation of prognostic factors.

\section{Conclusion}

This study showed that most COVID-19 deaths at the public reference hospital occurred due to severe acute respiratory syndrome, with a higher proportion of men, brown race and an average age of 60 years. The short time of hospital stay between hospitalization and death draws attention to the deficiency of the public health system of Rio de Janeiro city.

\section{Acknowledgments}

None.

\section{Conflicts of interest}

The authors declared no potential conflicts of interest with respect to the research, authorship, and/or publication of this article.

\section{Funding}

None.

\section{References}

1. Fehr AR, Perlman S. Coronaviruses: an overview of their replication and pathogenesis. Methods Mol Biol. 2015;1282:1-23.

2. Zhu N, Zhang D, Wang W, et al. A Novel Coronavirus from Patients with Pneumonia in China, 2019. N Engl J Med. 2020;382(8):727-733.

3. Shereen MA, Khan S, Kazmi A, et al. COVID-19 infection: Origin, transmission, and characteristics of human coronaviruses. J Adv Res. 2020;24:91-98.

4. Tay MZ, Poh CM, Rénia L, et al. The trinity of COVID-19: immunity, inflammation and intervention. Nat Rev Immunol. 2020;20(6):363-374.

5. Park M, Cook AR, Lim JT, et al. A Systematic Review of COVID-19 Epidemiology Based on Current Evidence. J Clin Med. 2020;9(4):967.

6. World Health Organization. Coronavirus disease (COVID-2019) situation reports. Geneva: World Health Organization; 2020.

7. Sontag AM, Franco C, Brandt DYC, et al. Observatório do COVID-19 BR. 2020.
8. Johns Hopkins Whiting School of Engineering. Center for Systems Science and Engineering. Coronavirus COVID19 Global Cases. 2020.

9. Loeffelholz MJ, Tang YW. Laboratory diagnosis of emerging human Coronavirus infections-The state of the art. Emerg Microbes Infect. 2020;9(1):747-756.

10. Cao X. COVID-19: Immunopathology and its implications for therapy. Nat Em Immunol. 2020;20(5):269-270.

11. Baxi P, Saxena SK. Emergence and Reemergence of Severe Acute Respiratory Syndrome (SARS) Corona viruses. Coronavirus Disease 2019 (COVID-19). 2020:51-163.

12. Huang C, Wang Y, Li X, et al. Clinical features of patients infected with 2019 novel coronavirus in Wuhan, China. Lancet. 2020;395(10223):497506.

13. Zhou F, Yu T, Du R, et al. Clinical course and risk factors for mortality of adult in patients with COVID-19 in Wuhan, China: a retrospective cohort study. Lancet. 2020;395(10229):1054-1062.

14. Lei S, Jiang F, Su W. Clinical characteristics and outcomes of patients undergoing surgeries during the incubation period of COVID-19 infection. EclinicalMedicine. 2020;21:100331.

15. Chen $\mathrm{T}, \mathrm{Wu} \mathrm{D}$, Chen $\mathrm{H}$, et al. Clinical characteristics of 113 deceased patients with coronavirus disease 2019: retrospective study. BMJ. 2020;368:m1091.

16. Salimi S, Hamlyn JM. COVID-19 and Crosstalk with the Hallmarks of Aging. J Gerontol A Biol Sci Med Sci. 2020;75(9):e34-e41.

17. Yao X, Li T, He Z. A pathological context three COVID-19 cases by minimally invasive autopsies. Chin J Pathol. 2020;49(5):E009.

18. Gianicolo E, Riccetti N, Blettner M, et al. Epidemiological Measures in the ontexto the COVID-19 Pandemic. Dtsch Arztebl Int. 2020;117(19):336342.

19. https://www.epicentro.iss.it/en/coronavirus/sars-cov-2-integratedsurveillance-data

20. Cavalcante JR, Abreu AJL. COVID-19 in the city of Rio de Janeiro: spatial analysis of first confirmed cases and deaths. Epidemiol Serv Saude. 2020;29(3):e2020204.

21. https://www.worldometers.info/coronavirus

22. Iyer LP, Ensor J, Anand K, et al. Higher mortality in men from COVID19 infection-understanding the factors that drive the differences between the biological sexes. medRxiv. 2020;20062174.

23. Richardson S, Hirsch JS, Narasimhan M. Presenting Characteristics, Comorbidities, and Outcomes Among 5700 Patients Hospitalized With COVID-19 in the New York City Area. JAMA. 2020;323(20):2052-2059.

24. Lauer SA, Grantz KH, Bi Q. The incubation period of coronavirus disease 2019 (COVID-19) from publicly reported confirmed cases: estimation and application. Ann Intern Med. 2020;172(9):577-582.

25. Cohen PA, Hall LE, John JN, et al. The Early Natural History of SARSCoV-2 Infection: Clinical Observations From na Urban, Ambulatory COVID-19 Clinic. Mayo Clin Proc. 2020;95(6):1124-1126.

26. Linton NM, Kobayashi T, Yang Y, et al. Incubation Period and Other Epidemiological Characteristics of 2019 Novel Coronavirus Infections with Right Truncation: A Statistical Analysis of Publicly Available Case Data. J Clin Med. 2020;9(2):538.

27. Goldwasser RS, Lobo MS, de Arruda EF. Difficulties in access and estimates of public beds in intensive care units in the state of Rio de Janeiro. Rev Saude Publica. 2016;50:19. 